\title{
Estimation of Markov-Modulated Time-Series via EM Algorithm
}

\author{
Subhrakanti Dey, Vikram Krishnamurthy, and Thierry Salmon-Legagneur
}

\begin{abstract}
In this letter, we consider the estimation of various Markov-modulated time series. We obtain maximum likelihood estimates of the time-series parameters including the Markov chain transition probabilities and the time-series coefficients using the expectation maximization (EM) algorithm. In addition, the recursive EM algorithm is used to obtain on-line parameter estimates. Simulation studies show that both algorithms yield satisfactory results.
\end{abstract}

\section{INTRODUCTION}

Signal Model: Let $s_{k}$ denote a $N_{s}$-state irreducible Markoy chain with states $\left\{1,2, \ldots, N_{s}\right\}$ with transition probability matrix $\Pi=\left(\pi_{m n}\right), \pi_{m n}=P\left(s_{k+1}=n \mid s_{k}=m\right)$ and initial state probability $\pi=\left(\pi_{m}\right), \pi_{m}=P\left(s_{1}=m\right)$. Define the Markov-modulated polynomials $A\left(z^{-1}, s_{k}\right), B\left(z^{-1}, s_{k}\right)$, and $C\left(z^{-1}, s_{k}\right)$ as (where $z^{-1}$ denotes the delay operator and $k$ denotes discrete time)

$$
\begin{aligned}
& A\left(z^{-1}, s_{k}\right)=1+\sum_{i=1}^{p} a_{i}\left(s_{k}\right) z^{-i} \\
& B\left(z^{-1}, s_{k}\right)=1+\sum_{i=1}^{q} b_{i}\left(s_{k}\right) z^{-i}, \\
& C\left(z^{-1}, s_{k}\right)=1+\sum_{i=1}^{r} c_{i}\left(s_{k}\right) z^{-i} .
\end{aligned}
$$

Let $A(m) \triangleq\left(a_{1}(m) \cdots a_{p}(m)\right)^{\prime}, B(m) \triangleq\left(b_{1}(m) \cdots b_{q}(m)\right)^{\prime}$, $C(m) \triangleq\left(c_{1}(m) \cdots c_{r}(m)\right)^{\prime}, m \in\left\{1,2, \ldots, N_{s}\right\}$.

In this letter, we consider parameter estimation of any one of the following second-order stationary (see Remark 4 below) Markov-modulated time-series models:

$$
\begin{aligned}
\text { ARX: } A\left(z^{-1}, s_{k}\right) y_{k} & =B\left(z^{-1}, s_{k}\right) u_{k}+w_{k} \\
\phi & =\left(A(m), B(m), \Pi, \sigma^{2}\right) \\
\text { MAX: } y_{k} & =B\left(z^{-1}, s_{k}\right) u_{k}+C\left(z^{-1}, s_{k}\right) w_{k} \\
\phi & =\left(B(m), C(m), \Pi, \sigma^{2}\right) \\
\text { ARMA: } A\left(z^{-1}\right) y_{k} & =C\left(z^{-1}, s_{k}\right) w_{k} \\
\phi & =\left(A, C(m), \Pi, \sigma^{2}\right)
\end{aligned}
$$

Manuscript received January 12, 1994; approved June 15, 1994. This work was supported by the Cooperative Research Centre for Robust and Adaptive Systems by the Australian Commonwealth Government under the Cooperative Research Centres Program. The associate editor coordinating the review of this letter and approving it for publication was Prof. T. S. Durrani.

S. Dey and V. Krishnamurthy are with the Cooperative Research Center for Robust and Adaptive Systems, Department of Systems Engineering, Research School of Information Sciences and Engineering, Australian National University, Canberra, Australia.

T. Salmon-Legagneur is with DSTO, Australia.

IEEE Log Number 9405625. where $u_{k}$ and $y_{k}$ are the measured input and output at time $k, w_{k} \sim$ white $N\left(0, \sigma^{2}\right)$ is independent of $s_{k}$, and $\phi$ is the parameter vector consisting of polynomial coefficients and Markov chain parameters. We assume $u_{k}$ to be persistently exciting [4]. We also assume that $A\left(z^{-1}, s_{k}\right), B\left(z^{-1}, s_{k}\right)$ and $C\left(z^{-1}, s_{k}\right)$ are coprime to each other for each $m, m \in$ $\left\{1,2, \ldots, N_{s}\right\}$.

Notations: $Y_{k}=\left(y_{1} \ldots y_{k}\right)^{T}, U_{k}=\left(u_{1} \cdots u_{k}\right)^{T}, S_{k}=$ $\left(s_{1} \cdots s_{k}\right)^{T}, Y_{t}^{k}=\left(y_{t} \cdots y_{k}\right)^{T}, U_{t}^{k}=\left(u_{t} \cdots u_{k}\right)^{T}$ and $Z_{k}=\left(Y_{k}, U_{k}\right)$, where superscript $T$ denotes transpose.

Estimation Objectives: We use the expectation maximization (EM) algorithm [10] to obtain maximum likelihood (ML) estimates of $\phi$, given $Y_{T}$ and $U_{T}$ (when appropriate) in Section II. In addition, based on the recursive EM algorithm [2], an on-line estimation scheme is presented in Section III.

Motivation and Applications: The models (1.2), (1.3), and (1.4) consist of parameter sets that are constant over segments with abrupt changes from segment to segment. The parameter sets are determined by the realization of a finite state Markov chain. Such so called "segmentation" models are used in econometrics, seismology, geology, and image analysis (see [5] and references therein).

In [5], the EM algorithm and a recursive EM algorithm are used to estimate Markov-modulated AR processes, which is a special case of our model (1.2) with $B=0$. The three models we consider in this paper can be regarded as an extension of the work in [5].

Our model can be also viewed as a random coefficient time series. These are used to model the stochastic stability of short run market equilibrium under variations in supply (see [9] and references therein). Markov-modulated models also used in econometrics [6] and failure detection [7].

Remark 1: Models (1.2), (1.3), or (1.4) are special cases of the Markov-modulated ARMAX model

$$
A\left(z^{-1}, s_{k}\right) y_{k}=B\left(z^{-1}, s_{k}\right) u_{k}+C\left(z^{-1}, s_{k}\right) w_{k} .
$$

However, unlike (1.2), (1.3), and (1.4), ML estimation of (1.5) is computationally prohibitive since it requires computing probability density functions over all $N_{s}^{T}$ realizations of a $N_{s}$ state $T$-point Markov chain. We do not deal with estimating (1.5) in this letter. For similar reasons, we forbid $A\left(z^{-1}\right)$ in (1.4) to be Markov modulated. Various suboptimal techniques for estimating Markov-modulated ARMAX models exist in the literature [11], [3].

Remark 2: As a more generalized model, one can have $A\left(z^{-1}, s_{k}\right), B\left(z^{-1}, t_{k}\right)$ and $C\left(z^{-1}, r_{k}\right)$ modulated by three independent Markov chains $s_{k}, t_{k}, r_{k}$. In this letter, we consider 
the special case $s_{k}=t_{k}=r_{k}$ for notational simplicity, although our approach can be easily extended. Another obvious extension is to consider polynomials $A\left(z^{-1}, S_{k}\right), B\left(z^{-1}, S_{k}\right)$, and $C\left(z^{-1}, S_{k}\right)$, where $S_{k}=\left(s_{k}, s_{k-1}, \ldots, s_{k-p}\right)^{\prime}$ is a vector state Markov chain.

Remark 3: State estimates of $s_{k}$ are obtained from the $E$ step of the EM algorithm.

Remark 4: Deriving stationarity criteria for Markovmodulated time series is a difficult problem. For example, two switching, separately second-order AR stationary processes can result in an unstable system-whereas two individually unstable AR processes can be stabilized when allowed to switch according to a Markov regime. For sufficient conditions on the second-order stationarity of Markov-modulated time series, see [8] and [5].

\section{- II. ML Estimation VIA EM ALgORITHM}

\section{A. Markov-Modulated ARX Estimation}

The EM algorithm for estimating $\phi$ in (1.2) involves two steps: E-step and M-step.

E Step: Following [3], the expectation of the log-likelihood function of a $T$-point "complete" data sequence $M_{T}=$ $\left(Y_{T}, U_{T}, S_{T}\right)$ defined as $\mathcal{Q}\left(\phi^{(l)}, \phi\right) \triangleq E\left\{\ln f\left(M_{T} \mid \phi\right) \mid Z_{T}\right.$, $\left.\phi^{(l)}\right\}$ can be written as

$$
\begin{aligned}
Q\left(\phi^{(l)}, \phi\right)= & -\frac{T}{2} \ln \sigma^{2}-\frac{1}{2 \sigma^{2}} \sum_{k=1}^{T-1} \sum_{m=1}^{N_{s}} \\
& \times \gamma_{k}(m)\left(A\left(z^{-1}, m\right) y_{k}-B\left(z^{-1}, m\right) u_{k}\right)^{2} \\
& +\sum_{k=1}^{T-1} \sum_{m=1}^{N_{s}} \sum_{n=1}^{N_{s}} \xi_{k}(m, n) \ln \pi_{m n} \\
& +\sum_{m=1}^{N_{s}} \gamma_{1}(m) \ln \pi_{m}
\end{aligned}
$$

where $\xi_{k}(m, n) \triangleq f\left(s_{k}=m, s_{k+1}=n \mid Z_{T}, \phi^{(l)}\right)$, and $\gamma_{k}(m) \triangleq f\left(s_{k}=m \mid Z_{T}, \phi^{(l)}\right) . \gamma_{k}(m)$ is computed via the "forward backward" procedure [1] as $\alpha_{k}(m) \beta_{k}(m) / \sum_{m=1}^{N_{s}} \alpha_{k}(m) \beta_{k}(m)$, where $\alpha_{k}(m)$ and $\beta_{k}(m)$ are calculated recursively as $\alpha_{k}(m)=\sum_{n=1}^{N_{s}} \alpha_{k-1}(n) a_{n m} b_{m}\left(y_{k}\right)$, and $\beta_{k}(m)=$ $\sum_{n=1}^{N_{s}} a_{m n} b_{n}\left(y_{k+1}\right) \beta_{k+1}(n)$.

Here $b_{m}\left(y_{k}\right) \triangleq f\left(y_{k} \mid Y_{k-p}^{k}, U_{k-q}^{k}, s_{k}=m, \phi\right)=$ $\frac{1}{\sqrt{2 \pi \sigma^{2}}} \exp \left(-\frac{\left(A\left(z^{-1}, m\right) y_{k}-B\left(z^{-1}, m\right) u_{k}\right)^{2}}{2 \sigma^{2}}\right) . \phi^{(l)}$ is the estimate of the parameter vector at the $l$ th iteration, assuming the iteration procedure starts with an initial estimate $\phi^{(0)}$.

$M$ Step: This step involves computing $\operatorname{argmax}_{\phi} Q\left(\phi^{(l)}, \phi\right)$. This yields

$$
\begin{aligned}
\pi_{m n}= & \frac{\sum_{k=1}^{T-1} \xi_{k}(m, n)}{\sum_{k=1}^{T-1} \gamma_{k}(m)} \\
\sigma^{2}= & \frac{1}{T-1} \sum_{k=1}^{T-1} \sum_{m=1}^{N_{s}} \gamma_{k}(m) \\
& \times\left(A\left(z^{-1}, s_{k}\right) y_{k}-B\left(z^{-1}, s_{k}\right) u_{k}\right)^{2} \\
A(m)= & R_{a}(m)^{-1} v_{a}(m),
\end{aligned}
$$

$$
\begin{aligned}
B(m)=R_{b}(j)^{-1} v_{b}(m), & \\
& m \in\left\{1,2, \ldots, N_{s}\right\}
\end{aligned}
$$

where $R_{a}(m) \in \Re^{p \times p}$ with elements $\sum_{k=1}^{T-1} \gamma_{k}(m) y(k-$ i) $y(k-j), i, j \in\{1,2, \ldots, p\} \quad R_{b}(m) \in \Re^{q \times q}$ with elements $\sum_{k=1}^{T-1} \gamma_{k}(m) u(k-i) u(k-j), i, j \in\{1,2, \ldots, q\}$ $v_{a}(n) \in \Re^{q}$ with elements $\sum_{k=1}^{T-1} \gamma_{k}(n)\left(y_{k}-B\left(z^{-1}\right.\right.$, $\left.\left.s_{k}\right) u_{k}\right) y_{k-i}, i \in\{1,2, \ldots, p\}$, and $v_{b}(n) \in \Re^{q}$ with elements $\sum_{k=1}^{T-1} \gamma_{k}(n)\left(A\left(z^{-1}, s_{k}\right) y_{k}-u_{k}\right) u_{k-i}, i \in\{1,2, \ldots, q\}$.

\section{B. Markov-Modulated MAX Estimation}

The MAX model (1.3) can be written in equivalent ARX form as $A^{\prime}\left(z^{-1}, s_{k}\right) y_{k}=B^{\prime}\left(z^{-1}, s_{k}\right) u_{k}+e_{k}$, where $A^{\prime}\left(z^{-1}, s_{k}\right)$ is "sufficiently" long enough (see Remark below) to ensure that $e_{k}$ is almost white and $B^{\prime}\left(z^{-1}, s_{k}\right)=$ $A^{\prime}\left(z^{-1}, s_{k}\right) B\left(z^{-1}, s_{k}\right)$. In addition, let $A^{\prime}(m)$ and $B^{\prime}(m)$ be the vectors containing the coefficients of $A^{\prime}\left(z^{-1}, s_{k}\right)$ and $B^{\prime}\left(z^{-1}, s_{k}\right), m \in\left\{1,2, \ldots, N_{s}\right\}$, respectively.

EM Algorithm: After estimating $A^{\prime}(m)$ and $B^{\prime}(m)$ by the above EM algorithm in Section II-A, $B(m)$ can be estimated by polynomial division. To estimate $C(m)$ in (1.3), a set of inverse Yule-Walker equations has to be solved (see p. 291 of [4]), which are

$$
\begin{aligned}
\theta_{t}(m)+\sum_{i=1}^{r} c_{i} \theta_{t-i}(m)=0 \quad & t \geq 1, \\
& m \in\left\{1,2 \ldots, N_{s}\right\}
\end{aligned}
$$

where $\theta_{t}(m), t \geq 1$ can be estimated from the coefficients of $A^{\prime}\left(z^{-1}, s_{k}\right)$ as $\theta_{t}(m)=\sum_{i=0}^{p^{\prime}-t} a_{i}^{\prime}(m) a_{i+t}^{\prime}(m)$, where $A^{\prime}\left(z^{-1}, s_{k}\right)=\sum_{i=0}^{p^{\prime}} a_{i}^{\prime}(m) z^{-i}, a_{0}^{\prime}=1$.

Remark: The order $p^{\prime}$ of $A^{\prime}\left(z^{-1}, s_{k}\right)$ has to be large enough to be a good approximation of $1 / C\left(z^{-1}, s_{k}\right)$ in (1.3) (see p. 291 of [4]). For rigorous details, see Theorem 8.3 .1 in p. 246 of [12], where it is proved that for weak consistency $p^{\prime}$ should be chosen as $O\left(T^{1 / 3}\right)$.

\section{Markov-Modulated ARMA Estimation}

Since $A$ in (1.4) is no longer Markov modulated, it can be estimated via the Yule-Walker equations

$$
\eta_{t}+\sum_{i=1}^{p} a_{i} \eta_{t-i}=0 \quad t \geq r+1
$$

where $\eta_{t}=E\left[y_{k} y_{k-t}\right]$ (see p. 289, [4]).

EM Algorithm: Rewrite (1.4) as $A\left(z^{-1}\right) A^{\prime}\left(z^{-1}, s_{k}\right) y_{k}=$ $e_{k}$, where $e_{k}$ and $A^{\prime}\left(z^{-1}, s_{k}\right)$ are as defined in Section II-B. After obtaining an estimate of $A\left(z^{-1}\right) A^{\prime}\left(z^{-1}, s_{k}\right)$ via EM, dividing $A\left(z^{-1}\right)$ by $A\left(z^{-1}\right) A^{\prime}\left(z^{-1}, s_{k}\right)$ gives an estimate of $C\left(z^{-1}, s_{k}\right)$, and hence, $C(m), m \in\left\{1,2, \ldots, N_{s}\right\}$.

Remark: $C\left(z^{-1}, s_{k}\right)$ could be also estimated by solving the inverse Yule-Walker equations (2.4) for $t \geq p+1$. However, simulations show that the above technique yields better results. 


\section{ON-LINE ESTIMATION VIA THE RECURSIVE EM ALGORITHM}

For brevity, we mention the relevant estimation equations only (for motivation and details of the recursive EM algorithm see [2] and the references therein).

Define $\phi_{k}$ as the estimate of the model $\phi$ at the $k$-th time instant and $\Phi_{k}=\left(\phi_{1}, \ldots, \phi_{k}\right)$. Following [2], our recursive EM algorithm based on maximizing the Kullback-Leibler information measure is

$$
\phi_{k+1}=\phi_{k}+\left(I_{k+1}\left(\phi_{k}\right)\right)^{-1} S\left(\phi_{k}, y_{k+1}\right)
$$

where $I_{k+1}\left(\phi_{k}\right)$ is the Fisher information matrix (FIM) of the complete data $M_{k}$, given by $I_{k+1}\left(\phi_{k}\right)=$ $-\partial^{2} \mathcal{Q}_{k+1}\left(\Phi_{k}, \phi\right) /\left.\partial \phi^{2}\right|_{\phi=\phi_{k}}, \mathcal{Q}_{k+1}\left(\Phi_{k}, \phi\right) \triangleq E\left\{\ln f\left(M_{k}\right.\right.$ $\left.\phi) \mid Z_{k}, \Phi^{(k-1)}\right\}$, and $S\left(\phi_{k}, y_{k+1}\right)$ is the score vector defined as $S\left(\phi_{k}, y_{k+1}\right)=\partial \mathcal{Q}_{k+1}\left(\Phi_{k}, \phi\right) /\left.\partial \phi\right|_{\phi=\phi_{k}}$.

\section{Remarks:}

1. Exponential forgetting can be used in updating the FIM as follows ( $\lambda_{F}=1$ means no forgetting):

$$
I_{k+1}=\lambda_{F} I_{k}+V_{k+1}, 0<\lambda_{F} \leq 1
$$

where $\lambda_{F}$ is the forgetting factor, and $V_{k+1}$ is that part of the FIM computed at time $k+1$.

2. In the ARMA estimation problem, $A\left(z^{-1}\right)$ is estimated by the recursive version of the Yule-Walker equations described in Section II with an appropriate forgetting factor $\lambda_{y w}$.

\section{Simulation Studies}

We present two examples, with $N_{s}=2, \pi_{11}=\pi_{22}=0.9$, and $u_{k}$ uniformly distributed in $(0,1)$ (where applicable).

\section{A. ML Estimation via EM Algorithm}

Results: For 50000 data-points, the following hold:

1. MAX: The true parameter vector $\phi_{0}=\left(B(1)^{\prime}, C(1)^{\prime}\right.$, $\left.B(2)^{\prime}, C(2)^{\prime}, \pi_{11} \pi_{22} \sigma^{2}\right)=\left(\begin{array}{ll}0.8 & 0.3\end{array}\right),\left(\begin{array}{ll}0.5 & 0.3\end{array}\right)$, $\left.\left(\begin{array}{lll}0.5 & 0.1\end{array}\right),\left(\begin{array}{lll}-0.4 & 0.2\end{array}\right), 0.90 .90 .25\right)$.

After 50 passes, we obtained $\phi^{50}=((0.78410 .2882)$, $\left(\begin{array}{lll}0.4756 & 0.2972\end{array}\right),\left(\begin{array}{lll}0.4740 & 0.1189\end{array}\right),\left(\begin{array}{lll}-0.3820 & 0.2043\end{array}\right)$, $\left(\begin{array}{lll}0.9066 & 0.9156 & 0.2586\end{array}\right)$.

2. ARMA: The true parameter vector $\phi_{0}=\left(A^{\prime}, C(1)^{\prime}\right.$, $\left.C(2)^{\prime}, \pi_{11} \pi_{22} \sigma^{2}\right)=((-1.00 .3),(0.50 .3),(-0.40 .2)$, $\begin{array}{lll}0.9 & 0.9 & 0.25)\end{array}$

$\phi^{50}=\left(\begin{array}{llll}(-1.0037 & 0.3078), & (0.4795 & 0.2892\end{array}\right)$,

$(-0.39550 .2162), 0.90990 .90870 .2525)$.

\section{B. On-Line Estimation via Recursive EM Algorithm}

Consider a jump time-varying 100000-point Markovmodulated ARMA model with $\sigma^{2}=1$ and

$$
\begin{gathered}
A=(0.8-0.5)^{\prime}, C(1)=(0.50 .3)^{\prime} \\
C(2)=(-0.40 .2)^{\prime} t \leq 20000 \\
A=(0.5-0.8)^{\prime}, C(1)=(0.70 .5)^{\prime} \\
C(2)=(-0.20 .5)^{\prime} t>20000 .
\end{gathered}
$$
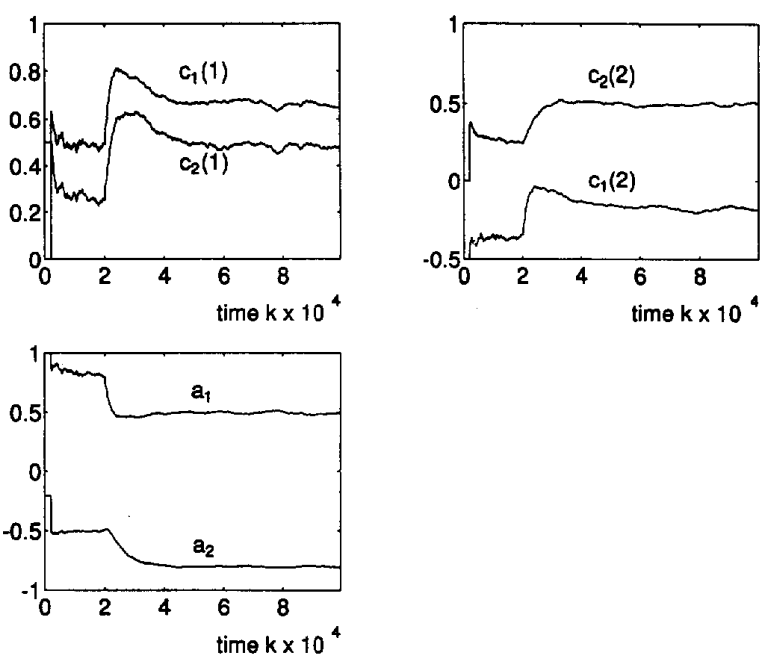

Fig. 1. Time evolution of ARMA parameter estimates.

In our simulation, $\lambda_{F}=0.9999, \lambda_{y w}=0.9999$, and $p^{\prime}=15$. We do not assume any a priori knowledge in the parameter values, and initial conditions may be arbitrary. To avoid the effect of the initial transients on the parameter estimates due to insufficient data, estimation starts after the first 2000 points, which is the period during which only the FIM is updated. Fig. 1 shows the time evolution of the estimates of the ARMA parameters.

Remark: The convergence proof of the recursive EM algorithm for HMM's is an open problem. Simulation studies show that the larger $\sigma^{2}$ is, the slower the convergence. It is seen from Fig. 1 that the estimates are close to the true values after a few thousand data points.

\section{REFERENCES}

[1] L. R. Rabiner, "A tutorial on Hidden Markov Models and selected applications in speech recognition," Proc. IEEE, vol. 77, no. 2, pp. 257-285, 1989.

[2] V. Krishnamurthy and J. B. Moore, "On-line estimation of hidden Markov model parameters based on the Kullback-Leibler information measure," IEEE Trans.Signal Processing, vol. 41, no. 8, pp. 2557-2573, Aug., 1993.

[3] D. M. Titterington, A. F. M. Smith and U. E. Makov, Statistical Analysis of Finite Mixture Distributions. New York: Wiley, 1985.

[4] T. Soderstrom and P. Stoica, System Identification. Englewood Cliffs, NJ: Prentice-Hall, 1989.

[5] U. Holst, G. Lindgren, J. Holst and M. Thuvesholmen, "Recursive estimation in switching autoregressions with Markov regime," to appear in J. Time-Series Anal., 1994.

[6] J. Hamilton, "Analysis of time-series subject to changes in regime," $J$ Econometrics, vol. 45 , pp. $39-70,1990$.

[7] M. Basseville and A. Benveniste, Detection of Abrupt Changes in Signals and Dynamical Systems. New York: Springer-Verlag, 1986.

[8] H. Karlssen, "A class of non-linear time series models," Ph.D. Thesis, Dept. of Math., Univ. of Bergen, Norway, 1990.

[9] D. F. Nicholls and B. G. Quinn, "The estimation of random coefficient autoregressive models. I," J. Time-Series Anal., vol. 1, no. 1, 1980, pp. $37-46$.

[10] A. P. Dempster, N. M. Laird, and D. B. Rubin, "Maximum likelihood from incomplete data via the EM algorithm," J. Royal Stat. Soc., ser. 39, vol. 6, pp. 1-38, 1977.

[11] V. Krishnamurthy, S. Dey and J. P. LeBlanc, "Blind equalisation of IIR channels using hidden Markov models," to appear in Proc. IEEE Int. Symp. Inform. Theory, Trondhiem, 1994.

[12] P. J. Brockwell and R. A. Davis, Time-Series: Theory and Methods. New York: Springer-Verlag, 1991, 2nd ed. 